\title{
ENVELHECIMENTO E ORDENAÇÃO TEMPORAL AUDITIVA
}

\section{Aging and auditory temporal resolution}

\author{
Flávia Duarte Liporaci ${ }^{(1)}$, Silvana Maria Monte Coelho Frota ${ }^{(2)}$
}

\begin{abstract}
RESUMO
Objetivo: avaliar o processamento auditivo em idosos através do teste de ordenação temporal (Padrão de Duração) e verificar se a presença de perda auditiva influencia no desempenho deste teste. Métodos: 65 idosos foram avaliados (46 mulheres e 19 homens), entre 60 e 79 anos. Os participantes responderam a uma anamnese, realizaram mini-exame do estado mental e avaliação audiológica básica. Os participantes foram alocados e estudados em um grupo único e também divididos em três grupos denominados G1, G2 e G3. De acordo com os resultados audiométricos, classificouse o G1 (audição normal para as médias de 0,5/1/2 kHz e de 3/4/6 kHz), o G2 (audição normal ou perda auditiva de grau leve para a média de $0,5 / 1 / 2 \mathrm{kHz}$ e grau leve para a média de3/4/6 kHz ) e o G3 (audição normal ou perda auditiva de grau leve para a média de 0,5/1/2 kHz e grau moderado para a média de3/4/6 kHz). Foi realizado o teste Padrão de Duração, que avalia a ordenação temporal, a 50dBNS. Para a análise dos resultados foi utilizado o teste estatístico não-paramétrico de KruskalWallis e o teste do qui-quadrado, sendo considerado como significante um $p<0,05$. Resultados: em toda a amostra a média de porcentagem de acertos foi de $63,1 \%$. Nos grupos, as médias de acertos foram de 57,5\%,69\% e 63,9\% para o G1, G2 e G3, respectivamente. Conclusão: a presença de perda auditiva não influenciou nos resultados do teste padrão de duração.
\end{abstract}

DESCRITORES: Percepção Auditiva; Idoso; Testes Auditivos; Transtornos da Percepção Auditiva

\section{INTRODUÇÃO}

$\mathrm{Na}$ área da audiologia, muitos estudos nacionais já foram realizados sobre a perda auditiva e o processo de envelhecimento ${ }^{1-4}$. No entanto, sabe-se que a audição decresce não só quantitativa, mas também qualitativamente, sendo comuns aos idosos as queixas de dificuldade em entender a linguagem falada, particularmente quando outras pessoas falam ao mesmo tempo, ou seja, quando há competição com mensagens de fundo. As causas subjacentes das dificuldades de percepção da fala nestes indivíduos ainda não estão claras ${ }^{5,6}$.

(1) Fonoaudióloga; Chefe do Serviço de Fonoaudiologia do Hospital Central da Marinha, HCM, Rio de Janeiro, RJ; Mestre em Fonoaudiologia pela Universidade Veiga de Almeida.

(2) Fonoaudióloga; Professora Adjunta do Curso de Fonoaudiologia da Universidade Federal do Rio de Janeiro, UFRJ, Rio de Janeiro, RJ; Professora Adjunta do Curso de Mestrado em Fonoaudiologia da Universidade Veiga de Almeida, UVA, Rio de Janeiro, RJ; Doutora em Distúrbios da Comunicação Humana pela Universidade Federal de São Paulo.

Conflito de interesses: inexistente
O uso da audiometria tonal e vocal, para a avaliação da audição do idoso, parece não ser suficiente para prover informações a respeito de "como" e "quanto" a perda auditiva influencia a sua comunicação, pois a percepção que ele tem de sua capacidade de ouvir é muitas vezes pior do que o esperado por seu audiograma.

A avaliação do processamento auditivo verifica como o indivíduo recebe as informações acústicas utilizando habilidades auditivas que são essenciais para ele apreender o que ouve. No conjunto de testes da avaliação do processamento auditivo a análise dos processos temporais deve constar.

As mudanças acústicas que ocorrem dentro de um tempo parecem ser a essência da percepção auditiva temporal; um intervalo entre dois sons de 15 a $20 \mathrm{~ms}$ é necessário para que o ouvinte perceba qual dos dois sons precedeu o outro. A duração deste intervalo sugere que a capacidade de analisar a ordenação de sons requer mecanismos que não são somente associados com o sistema auditivo periférico, mas também com estruturas centrais. Para percepção da fala, sons não são somente diferenciados um do outro, mas também 
devem ser avaliados com respeito a sua ordem de ocorrência ${ }^{7}$.

Muitas características, se não todas, que envolvem a informação auditiva, são de alguma maneira influenciadas pelo tempo. A ordenação temporal refere-se ao processamento de dois ou mais estímulos acústicos na sua ordem de ocorrência no tempo. A habilidade de reconhecer corretamente, identificar, e seqüenciar padrões auditivos envolve muitos processos cognitivos e perceptivos; a memória de curto prazo parece estar envolvida ${ }^{8}$.

O envelhecimento pode trazer um declínio na habilidade de ordenação temporal que pode estar relacionado à redução da efetividade da comunicação. A presença de perda auditiva, frequente nesta fase da vida ${ }^{1-4}$, pode prejudicar ainda mais 0 desempenho em testes de processamento auditivo.

O objetivo deste estudo foi avaliar o processamento auditivo em idosos por meio do teste de ordenação temporal Padrão de Duração (PD) e verificar se a presença de perda auditiva influencia no desempenho deste teste.

\section{MÉTODOS}

Foi realizado estudo clínico prospectivo, selecionando indivíduos idosos que procuraram o Serviço de Fonoaudiologia do Hospital Central da Marinha, na cidade do Rio de Janeiro, no período de maio a setembro de 2008.

Para selecionar a amostra foram realizados: anamnese, otoscopia, mini-exame do estado mental ${ }^{9}$ e avaliação audiológica básica.

Foram incluídos somente indivíduos alfabetizados e com ausência de histórico de: cirurgias otológicas; distúrbios neurológicos; exposição a ruído ocupacional/trauma acústico; prática musical sistemática; alterações otológicas e uso crônico de medicamentos psicotrópicos nos últimos 12 meses. A ausência de obstrução do meato acústico externo na otoscopia também foi fator de inclusão.

No mini-exame do estado mental foram incluídos somente os indivíduos que obtiveram uma pontuação igual ou maior do que 24 .

Os indivíduos que concordaram em participar da pesquisa assinaram o termo de consentimento livre e esclarecido.

A amostra foi composta por 65 idosos, de ambos os sexos (46 mulheres e 19 homens), com idades entre 60 e 79 anos.

Os resultados da audiometria tonal serviram de base para alguns fatores de inclusão descritos a seguir. Quando houve perda auditiva, foram incluídos somente os indivíduos com perdas do tipo neurossensorial, sendo que para estes, as perdas deveriam ser simétricas, a fim de evitar que perdas auditivas assimétricas interferissem nos resultados dos procedimentos da pesquisa. Foram incluídos os indivíduos que tiveram, na média dos limiares auditivos das frequências baixas e médias $(500 \mathrm{~Hz}$, $1000 \mathrm{~Hz}$ e $2000 \mathrm{~Hz}$ ), audição normal ou perda de grau leve, ou seja, média de até $40 \mathrm{~dB}$ NA, e os que tiveram, na média das frequências altas (3000 Hz, $4000 \mathrm{~Hz}$ e $6000 \mathrm{~Hz}$ ), audição normal, perda de grau leve ou de grau moderado, ou seja, média de até $55 \mathrm{~dB}$ NA. Neste estudo foi incluído o cálculo da média dos limiares tonais das frequências altas $(3000 \mathrm{~Hz}, 4000 \mathrm{~Hz}$ e $6000 \mathrm{~Hz})$. Então, para estabelecer o grau da perda auditiva, foram adotadas as médias dos limiares tonais das frequências baixas e médias $(500 \mathrm{~Hz}, 1000 \mathrm{~Hz}$ e $2000 \mathrm{~Hz}$ ), e também das frequências altas $(3000 \mathrm{~Hz}, 4000 \mathrm{~Hz}$ e $6000 \mathrm{~Hz}$ ). Foi utilizado o audiômetro AMPLAID A-177 calibrado segundo a norma ISO 8253-1.

Quando a média das frequências baixas e médias foi diferente da média das frequências altas em uma mesma orelha, classificou-se o grau da perda auditiva considerando a pior média. Para estabelecer o grau da perda do indivíduo foi adotado um critério semelhante ao descrito anteriormente. Quando houve diferença entre o grau da perda da orelha direita e o grau da perda da orelha esquerda, a classificação pior foi adotada.

A partir da classificação quanto ao grau da perda auditiva, utilizando os critérios supracitados, os indivíduos foram dispostos em três grupos distintos designados neste estudo de G1, G2 e G3. O G1 com audição normal em ambas as médias (frequências baixas/médias e frequências altas), o G2 com audição normal ou perda leve para as frequências baixas/médias e com perda leve para as frequências altas; e o G3 com audição normal ou perda leve para as frequências baixas/médias e com perda moderada para as frequências altas (Figura1).

$\mathrm{Na}$ imitanciometria, cujo equipamento utilizado foi o GSI-38, foram incluídos aqueles com timpanogramas com curvas do tipo "A", "Ad" e "Ar", e com reflexos presentes no modo contra-lateral em pelo menos duas frequências.

Os indivíduos foram estudados como um grupo único $(n=65)$ e separados em subgrupos, conforme classificação já referida anteriormente: 26 indivíduos no G1, 22 indivíduos no G2 e 17 indivíduos no G3.

O teste padrão de duração ${ }^{10}$, gravado em $C D$, foi aplicado por meio de audiômetro acoplado a $C D$ player PHILIPS AZ7363, em cabina acústica. Ele foi apresentado ao participante a $50 \mathrm{~dB}$ NS acima da média aritmética dos limiares tonais das frequências de: 500, 1000 e $2000 \mathrm{~Hz}$ da pior orelha. Os estímulos foram apresentados de forma binaural. 


\begin{tabular}{|ccc|}
\hline GRUPOS & MÉDIA 500, 1000 e 2000 Hz & MÉDIA 3000, 4000, e 6000 Hz \\
\hline G1 & Normal (até $25 \mathrm{~dB})$ & Normal (até $25 \mathrm{~dB})$ \\
G2 & Normal ou leve $($ até $40 \mathrm{~dB})$ & Leve $(26$ a $40 \mathrm{~dB})$ \\
G3 & Normal ou leve (até $40 \mathrm{~dB})$ & Moderada $(41$ a $55 \mathrm{~dB})$ \\
\hline
\end{tabular}

Figura 1 - Distribuição dos grupos de acordo com o grau da perda auditiva

O teste é composto por 60 itens, cada um com uma sequência de três tons puros de $1 \mathrm{kHz}$, que se diferenciam quanto à duração: longo $(\mathrm{L})$ e curto (C). O tom longo dura $500 \mathrm{~ms}$ e o tom curto 250 $\mathrm{ms}$. O intervalo entre os tons é de $300 \mathrm{~ms}$. São seis possibilidades de sequências: LLC; LCL; LCC; CLL; CLC e CCL. Foram utilizadas as três sequências iniciais para treino e as 45 seguintes para a coleta de dados ${ }^{11}$. O participante, após ouvir cada sequência, nomeou os estímulos ouvidos, como por exemplo: longo-longo-curto. Só foi considerada como acerto a sequência que teve seus três tons nomeados corretamente. Os resultados foram anotados em uma folha de registro específica e os acertos pontuados em percentual.

Este estudo foi submetido à apreciação do Comitê de Ética em Pesquisa do Hospital Naval Marcílio Dias, sob o protocolo ${ }^{\circ}$ 04.3.2008.

Foram utilizados métodos não-paramétricos, pois as variáveis não apresentaram distribuição normal Gaussiana devido à dispersão dos dados e/ ou falta de simetria da distribuição.

Para a comparação de variável categórica (sexo) entre os grupos, foi aplicado o teste do quiquadrado $\left(c^{2}\right)$.

Para a comparação das medidas entre os três grupos estudados no teste PD, foi realizada a Análise de Variância de Kruskal-Wallis (ANOVA não-paramétrica). O teste de comparações múltiplas não paramétrico baseado na estatística de
Kruskal-Wallis foi aplicado para identificar quais os grupos que diferiram entre si, ao nível de $5 \%$.

O nível de significância deste estudo foi definido em $0,05(5 \%)$.

\section{RESULTADOS}

Com relação à faixa-etária dos indivíduos, a média de idade na amostra geral foi de $67,3( \pm 4,6)$ anos, variando de 60 a 79 anos.

Com base no $p$-valor, houve diferença estatisticamente significante na média de idade entre os grupos. A média de idade aumentou do G1 (65,9 anos) para o G2 (67,5 anos), e do G2 para o G3 (69,4 anos). Pelo teste de comparações múltiplas, identificou-se, ao nível de 5\%, que o G3 apresentou média de idade significantemente maior que o G1.

A amostra geral foi constituída por indivíduos de ambos os sexos, sendo que $70,8 \%$ era do sexo feminino.

Observou-se, segundo o teste de $c^{2}$, que não existiu diferença estatisticamente significante ( $p$-valor $=0,35)$ na proporção de mulheres entre os grupos G1 (80,8\%), G2 (63,6\%) e G3 (64,7\%).

A Tabela 1 apresenta os valores médios das porcentagens de acertos do PD na amostra geral e em cada grupo.

Observa-se que não houve diferença estatisticamente significante nos resultados do teste PD entre os grupos, ao nível de $5 \%$.

Tabela 1 - Média das porcentagens de acertos do PD na amostra geral e nos grupos

\begin{tabular}{cccccccc}
\hline PD & $\mathbf{n}$ & Média & Mediana & DP & Mínimo & Máximo & p-valor a \\
\hline AG & 65 & 63,1 & 66,7 & 25,4 & 13,3 & 100 & - \\
\hline G1 & 26 & 57,5 & 56,7 & 25,6 & 22,2 & 100 & \\
G2 & 22 & 69,0 & 74,5 & 24,9 & 24,4 & 100 & 0,29 \\
G3 & 17 & 63,9 & 68,9 & 25,4 & 13,3 & 91,1 & \\
\hline
\end{tabular}

Legenda:

$A G=$ amostra geral

$\mathrm{n}=$ número de indivíduos

DP: Desvio Padrão

a ANOVA de Kruskal-Wallis 


\section{DISCUSSÃO}

O processamento temporal é o componente fundamental da maioria das habilidades de processamento auditivo, entretanto, seus mecanismos neurais subjacentes não são bem compreendidos. Ele pode ser observado em muitos níveis, variando desde o nível mais básico de regulação de tempo neural no nervo auditivo até o processamento cortical para a audição binaural e percepção da fala ${ }^{11}$.

A ordenação temporal refere-se ao processamento de dois ou mais estímulos auditivos na sua ordem de ocorrência dentro de um período de tempo. Nesta habilidade tão importante para a linguagem estão envolvidos os dois hemisférios cerebrais. $O$ reconhecimento do contorno do estímulo auditivo é processado pelo hemisfério direito e transferido, via corpo caloso, ao hemisfério esquerdo, onde a rotulação linguística é aplicada ao estímulo 8,11,12.

Nesta pesquisa, a média de idade de toda a amostra foi de 67,3 anos, porém ela foi diferente para cada grupo. O G3, que foi constituído por indivíduos com perdas auditivas de grau moderado, teve a média de idade maior que o G2, com perdas auditivas de grau leve. Por sua vez, o G2 teve média de idade maior que o G1, constituído por indivíduos com audição normal.

Fazendo a comparação dos grupos entre si, foi visto que houve diferença estatisticamente significante, ou seja, quanto maior a média de idade, maior a severidade da perda auditiva encontrada. Outros estudos, assim como o atual, também mostraram a evolução da perda auditiva com o aumento da idade ${ }^{2,4}$.

Em relação ao fator gênero, os participantes foram, em sua maioria, do sexo feminino, fato que também ocorreu em outras pesquisas com idosos ${ }^{2,13-16}$. E, ao ser feita a análise da constituição de cada grupo quanto ao gênero, verificou-se que não houve diferença estatisticamente significante na proporção de mulheres entre os grupos, sendo assim, nos três grupos houve prevalência do sexo feminino.

Se for observada a porcentagem de participantes de cada grupo, pode-se verificar que o maior grupo é o de indivíduos com audição normal (G1), isto provavelmente tem relação com os critérios de exclusão utilizados para os portadores de perdas auditivas, tais como a assimetria entre as orelhas e o grau de severidade.

Originalmente, este teste é aplicado de forma monoaural, utilizando 30 estímulos para cada orelha. No decorrer dos anos algumas pesquisas não encontraram diferenças nos resultados segundo a variável orelha ${ }^{10,17,18}$. A literatura atual ${ }^{11}$ aponta que ele pode ser utilizado de forma binaural e com 45 itens, como realizado por esta pesquisa, sem que houvesse comprometimento de sua sensibilidade e especificidade. É importante ressaltar que não encontramos na literatura estudos que tivessem utilizado esta mesma metodologia.

Analisando as respostas da amostra geral, verifica-se que a média de acertos $(63,1 \%)$ foi bem menor do que a encontrada em adultos jovens com audição normal $(83 \%){ }^{17} \mathrm{e}$, mais próxima da média encontrada em outro estudo com idosos com audição normal $(67,5 \%){ }^{18}$. Desta forma, pode-se inferir que o envelhecimento traz prejuízo à habilidade de ordenação temporal pela diferença existente entre esta pesquisa e a pesquisa com adultos jovens ${ }^{17}$, e pela semelhança de resultados com a pesquisa com idosos ${ }^{18}$.

A amostra geral da pesquisa atual foi composta por idosos com e sem perda auditiva, diferentemente da pesquisa com idosos com audição normal referida anteriormente ${ }^{18}$. Então, pode ser observado que apesar da existência de idosos com perda auditiva até o grau moderado, foram encontrados resultados relativamente semelhantes com os da pesquisa citada com idosos com audição normal. Isto vem ao encontro à literatura estudada que apontou que o teste padrão de duração não se mostrou influenciado por perdas auditivas cocleares de grau leve a moderado ${ }^{10,16}$.

Ao ser examinado detalhadamente os resultados da amostra geral deste estudo, é possível notar que ela teve o mínimo, o máximo e o desvio padrão com valores bem próximos aos encontrados em outra pesquisa com idosos ${ }^{18}$, ou seja, valores que mostram uma certa variabilidade nas respostas. Tal fato constitui uma informação relevante quanto à questão de ser um teste que não tem muita consistência com as respostas em idosos. De certa forma, isso pode levantar a hipótese de que outros fatores ligados ao envelhecimento estejam relacionados a esta variação de respostas, uma vez que já foi visto que a perda auditiva não influenciou.

Ao serem averiguados os resultados de cada grupo separadamente, constata-se que as medidas de dispersão confirmam a variabilidade de respostas em idosos. Pode ser realizada a comparação do G1, composto por 26 idosos com audição normal, com idade média de 65,9 anos, com o grupo estudado em pesquisa semelhante ${ }^{18} \mathrm{com} 25$ idosos com audição normal, com idade média de 67,4 anos. No primeiro houve uma porcentagem média de acertos de $57,5 \%$ e no segundo de $67,5 \%$.

Então, como esse teste não é influenciado pela presença de perda auditiva ${ }^{10}$ e pela variação do nível de intensidade de aplicação ${ }^{17}$, talvez seja 
uma importante ferramenta para a avaliação de deficientes auditivos.

Partindo para a comparação intergrupos, verifica-se que não existiu diferença estatisticamente significante entre os grupos estudados no desempenho do teste. Sendo assim, o agravamento do grau da perda auditiva não influenciou de forma significante nos resultados.

Nesta pesquisa, não se pode afirmar que os fatores perda auditiva e idade influenciaram na habilidade de ordenação temporal. Salienta-se que ao ser colocado que a idade não influenciou nesta habilidade, isso se refere à variação da faixa etária que houve dentro desta pesquisa (60 a 79 anos), mesmo porque, já foi abordada a diferença destes resultados com os de pesquisa com adultos jovens.

É importante refletir sobre aspectos do envelhecimento que podem contribuir para o declínio nas respostas deste teste e, também, sobre a razão para os dados dispersos que foram encontrados, uma vez que as porcentagens de acertos variaram de forma considerável. Sendo assim, serão discutidos a seguir alguns fatores que podem ter influenciado nos resultados obtidos na presente pesquisa.

Interações complexas ocorrem entre as operações sensoriais e as cognitivo-linguísticas de ordem superior, de forma simultânea e sequencial por meio do sistema nervoso auditivo central. A codificação neurofisiológica dos sinais auditivos do nervo auditivo até o cérebro, referida como bottom-up, denota os mecanismos e processos que ocorrem no sistema auditivo periférico até as operações linguísticas e cognitivas de ordem superior em nível cortical. Se a codificação bottom-up dos sinais auditivos sofre algum dano em qualquer ponto ao longo das vias auditivas centrais, a percepção auditiva final será afetada. Vale lembrar que os fatores bottom-up são influenciados por fatores de ordem superior, tais como a atenção, a memória e a competência linguística, através de complexos mecanismos de alimentação e retroalimentação. Isto é, o cérebro não é organizado como um sistema meramente hierárquico, no qual a informação se dirige somente em uma direção e é processada sequencialmente em níveis ascendentes do sistema nervoso central. Mais do que isso, existem múltiplas representações da informação sensorial através do sistema e cada área é conectada a muitas outras ${ }^{12}$.

A resposta correta na realização deste teste exige a memória dos três tons ouvidos para a posterior evocação. Sabe-se que a memória está presente no processamento auditivo ${ }^{19}$; então, há a hipótese de que alguns idosos podem ter tido respostas piores, não porque não conseguiram discriminar o tom longo do tom curto, mas porque poderiam ter algum prejuízo na memória. Apesar de termos realizado o mini-exame do estado mental ${ }^{9}$, algumas dificuldades podem não ter sido identificadas por este instrumento de triagem. Alguns autores ${ }^{8}$ também referiram que a memória de curto prazo parecia estar envolvida no processo de ordenação temporal.

A amostra desta pesquisa foi constituída com idosos com vários níveis de escolaridade. Apesar deste estudo não ter tido o objetivo de comparar o nível de escolaridade com o desempenho no teste, parece não ter havido uma relação consistente entre estes dados, ou seja, houve participantes com $2^{\circ}$ grau e com nível superior que tiveram grande dificuldade na realização deste teste, e outros somente com o primário, que tiveram bom desempenho. Porém, são necessárias novas pesquisas para constatar tal observação. Em pesquisa realizada recentemente ${ }^{20}$, foi levantada a hipótese de que o nível educacional poderia contribuir para o declínio relacionado ao envelhecimento observado na tarefa de ordenação temporal. Os autores da referida pesquisa salientam que importantes transformações ocorrem no sistema educacional através das gerações, tornando impossível balancear grupos etários de idosos no que diz respeito à educação.

Por se tratar de tarefa psicoacústica, este teste requer uma resposta comportamental consciente do indivíduo, que pode sofrer efeito de muitos fatores, como a atenção. Este teste foi o último procedimento realizado durante a pesquisa. Como o indivíduo ficava cerca de 180 minutos sendo avaliado, há a possibilidade de que o desempenho tenha sido afetado em virtude do declínio dos níveis de atenção, concentração ou motivação no final da sessão. Sabe-se que as respostas neurais do córtex auditivo não dependem somente dos estímulos acústicos, a regulação da atenção presumivelmente desempenha uma importante função de bloqueio para a informação perceptiva ${ }^{21}$. Enfim, o estímulo acústico de entrada pode ser influenciado por fatores de ordem superior, como a atenção, a memória e a competência linguística, através de complexos mecanismos de alimentação e retroalimentação ${ }^{12}$.

A forma de resposta de um teste psicoacústico também pode influenciar nos resultados ${ }^{22}$. Talvez se as respostas tivessem sido do tipo murmurada os resultados fossem melhores, uma vez que outra pesquisa que comparou a resposta por nomeação com a resposta do tipo murmurada encontrou maior porcentagem de acertos nesta última modalidade ${ }^{17}$. As dificuldades apresentadas na atual pesquisa nas respostas por nomeação podem exprimir alteração no processo de transferência inter-hemisférica ${ }^{8,11}$, uma vez que o reconhecimento do contorno do padrão ocorre no hemisfério 
direito e a informação é transferida através do corpo caloso para o hemisfério esquerdo, onde a nomeação lingüística do sinal é feita. Sendo assim, nos casos onde há respostas verbais insatisfatórias, o déficit auditivo perceptivo não pode ser presumido; nesses casos é mais provável que haja uma disfunção na transferência inter-hemisférica para o hemisfério esquerdo.

Como já referido, a tarefa deste teste requisita que haja transferência inter-hemisférica, ou seja, os dois hemisférios estão envolvidos. Outra hipótese sobre o pior desempenho em idosos, quando comparado com jovens, seria o efeito do envelhecimento nas assimetrias hemisféricas. Este efeito foi estudado em uma pesquisa que constatou, através de exames objetivos, que o padrão de dominância esquerda visto nas crianças e nos adultos jovens não é evidente nos adultos mais velhos, podendo ser um fator que contribua para as dificuldades de processamento temporal. Com o envelhecimento as respostas hemisféricas tornam-se mais simétricas ${ }^{22}$. Desta forma, neste teste, infere-se que o déficit no hemisfério esquerdo em idosos possa ter levado a alterações no processamento da informação e na rotulação dos estímulos acústicos vindos do hemisfério direito.

A evocação de ordenação temporal para seqüências de três tons, como no teste utilizado na presente pesquisa, é significantemente pior para ouvintes idosos do que para ouvintes jovens ${ }^{23}$. Diante disso, a normatização de um teste de ordenação temporal com dois tons para idosos pode ser importante como instrumento de avaliação dessa população. Ademais, foi observado durante a aplicação deste teste, que a maior parte dos participantes percebia quando não estava tendo um bom desempenho, e isto pareceu interferir de forma negativa no transcurso do teste. Apesar de não haver meios de medir tal interferência, talvez um teste com menor complexidade seja eficaz para manter um bom nível de motivação durante a aplicação.

Por fim, é importante que a mesma metodologia desta pesquisa, quanto ao modo de apresentação dos estímulos e ao número de itens, seja utilizada em outros estudos para uma melhor comparação de resultados.

A despeito deste estudo não ter como objetivo comparar o desempenho de idosos com jovens, ele traz um recorte do desempenho de uma população de idosos em habilidades do processamento temporal auditivo e, ao ser relacionado a estudos encontrados na literatura com adultos jovens ${ }^{10,17}$, mostra um declínio nesta habilidade.

A avaliação da audição do idoso, através da audiometria tonal e do exame do processamento auditivo, deve passar a fazer parte da rotina de orientação do profissional da área médica. Os resultados desta pesquisa estão de acordo com estudos que foram publicados ${ }^{5,13,14,16,18,20,24-26}$ associando o envelhecimento às alterações no processamento auditivo; logo, o declínio na função auditiva central tem valor suficiente e não deve ser ignorado.

Estudos envolvendo o treinamento das habilidades dos aspectos temporais da audição nesta população devem ser realizados, pois poderão trazer ganhos na qualidade da comunicação e acrescentar informações importantes para o processo de reabilitação auditiva.

\section{CONCLUSÃO}

Quanto à avaliação da habilidade de ordenação temporal através do teste de Padrão de Duração:

$\mathrm{Na}$ amostra geral a média de porcentagem de acertos foi de $63,1 \%$; e

A presença de perda auditiva não influenciou na porcentagem de acertos. 


\section{ABSTRACT}

Purpose: to evaluate the auditory processing in elderly through temporal resolution (Duration Pattern) test and to check if the presence of hearing loss influences the performance of this test. Methods: 65 elderly listeners were evaluated (46 women and 19 men), with ages ranging from 60 and 79 year old. All of them answered an anamnesis and passed through a mini mental state examination and basic audiological evaluation. The participants were placed and studied in a single group and also divided into three groups referred to as G1, G2 and G3. According to the audiometric results we classified the G1 (normal hearing for the averages of $0.5 / 1 / 2 \mathrm{kHz}$ and $3 / 4 / 6 \mathrm{kHz}$ ), the $\mathrm{G} 2$ (normal hearing or mild hearing loss for the average of $0.5 / 1 / 2 \mathrm{kHz}$ and mild hearing loss for the average of $3 / 4 / 6 \mathrm{kHz}$ ) and the G3 (normal hearing or mild hearing loss for the average of $0.5 / 1 / 2 \mathrm{kHz}$ and moderate hearing loss for the average of $3 / 4 / 6 \mathrm{kHz}$ ). The Duration Pattern Test that evaluates the temporal resolution was carried out at $50 \mathrm{dBSL}$. For analyzing the results we used the non-parametric statistical test of KruskalWallis and Chi - square, considering a significant $p<0.05$. Results: in the whole sample, the average of percentage concerning correct responses was $63.1 \%$. In the groups, the average percentage of correct responses was $57.5 \%, 69 \%$ and $63.9 \%$ to $\mathrm{G} 1, \mathrm{G} 2$ and G3, respectively. Conclusion: the presence of hearing loss did not influence the results of the Duration Pattern Test.

KEYWORDS: Auditory Perception; Aged; Hearing Tests; Auditory Perceptual Disorders

\section{REFERÊNCIAS}

1. Pinzan-Faria VM, lório MCM. Sensibilidade auditiva e autopercepção do handicap: um estudo em idosos. Dist Comun. 2004; 16(3):289-99.

2. Baraldi GS, Almeida LC, Borges ACC. Evolução da perda auditiva no decorrer do envelhecimento. Rev. Bras. Otorrinolaringol. 2007; 73(1):64-70.

3. Mattos LC, Veras RP. Prevalência da perda auditiva em uma população de idosos da cidade do Rio de Janeiro: um estudo seccional. Rev. Bras. Otorrinolaringol. 2007; 73(5):654-9.

4. Sousa MGC, Russo ICP. Audição e percepção da perda auditiva em idosos. Rev Soc Bras Fonoaudiol. 2009; 14(2):241-6.

5. Phillips SL, Gordon-Salant S, Fitzgibbons PJ, Yeni-Komshian G. Frequency and temporal resolution in elderly listeners with good and poor word recognition. J Speech Lang Hear Res. 2000; 43(1):217-28.

6. Bellis TJ. Differential diagnosis of (central) auditory processing disorder in older listeners. In: Musiek FE, Chermak GD, organizadores. Handbook of (central) auditory processing disorder: auditory neuroscience and diagnosis volume I. San Diego: Plural Publishing; 2007. p. 319-46.

7. Hirsh IJ. Auditory perception of temporal order. J Acoust Soc Am. 1959; 31(6):759-67.

8. Pinheiro M, Musiek E. Assessment of central auditory dysfunction: Foundations and clinical correlates. Baltimore: Williams \& Wilkins; 1985.
9. Brucki SMD, Nitrini R, Caramelli P, Bertolucci PHF, Okamoto IH. Sugestões para o uso do mini-exame do estado mental no Brasil. Arq Neuropsiquiatr. 2003; 61(3B):777-81.

10. Musiek FE, Baran JA, Pinheiro ML. Duration pattern recognition in normal subjects and patients with cerebral and cochlear lesions. Audiology. 1990; 29:304-13.

11. Shinn JB. Temporal processing and temporal patterning tests. In: Musiek FE, Chermak GD, organizadores. Handbook of (central) auditory processing disorder: auditory neuroscience and diagnosis. vol 1. San Diego: Plural Publishing; 2007. p. 231-56.

12. Bellis TJ. Assessment and management of central auditory processing disorders in the educational setting from science to practice. 2. ed. Clifton Park, NY: Singular/Delmar Learning; 2003. p. 50-102.

13. Quintero SM, Marotta RMB, Marone SAM. Avaliação do processamento auditivo de indivíduos idosos com e sem presbiacusia por meio do teste de reconhecimento de dissílabos em tarefa dicótica - SSW. Rev. Bras. Otorrinolaringol. 2002; 68(1):28-33.

14. Geal-Dor M, Goldstein A, Kamenir Y, Babkoff $\mathrm{H}$. The effect of aging on event-related potentials and behavioral responses: comparison of tonal, phonologic and semantic targets. Clin Neurophysiol. 2006; 117:1974-89.

15. Calais LL, Borges ACLC, Baraldi GS, Almeida LC. Queixas e preocupações otológicas e as 
dificuldades de comunicação de indivíduos idosos. Rev Soc Bras Fonoaudiol. 2008; 13(1):12-9.

16. Azzolini VC, Ferreira MID. Processamento Auditivo Temporal em Idosos. Arq. Int. Otorrinolaringol.2010;14(1):95-102.

17. Corazza MCA. Avaliação do processamento auditivo central em adultos: testes de padrões tonais auditivos de frequência e teste de padrões tonais auditivos de duração [tese]. São Paulo (SP): Universidade Federal de São Paulo; 1998.

18. Parra VM, lório MCM, Mizahi MM, Baraldi GS. Testes de padrão de freqüência e de duração em idosos com sensibilidade auditiva normal. Rev Bras Otorrinolaringol. 2004; 70(4):517-23.

19. Miranda ES, Pereira LD, Bommarito S, Silva TM. Avaliação do processamento auditivo de sons não-verbais em indivíduos com doença de Parkinson. Rev Bras Otorrinolaringol. 2004; 70(4):534-9.

20. Kolodziejczyk I, Szelag E. Auditory perception of temporal order in centenarians in comparison with Young and elderly subjects. Acta Neurobiol Exp. 2008; 68:373-81.
21. Ruytjens L, Willemsen ATM, Van Dijk P, Wit HP, Albers FWJ. Functional imaging of the central auditory system using PET. Acta Otolaryngol. 2006; 126(12):1236-44.

22. Bellis TJ, Nicol T, Kraus, N. Aging affects hemispheric asymmetry in the neural representation of speech sounds. J Neuroscience. 2000; 20(2):791-7.

23. Gordon-Salant S. Speech perception and auditory temporal processing performance by older listeners: implications for real-world communication. Semin Hear. 2006; 27:264-8.

24. Roberts RA, Lister JJ. Effects of age and hearing loss on gap detection and the precedence effect: broadband stimuli. J. speech lang. hear. res. 2004;47:965-78.

25. Grose JH, Hall JW, Buss E. Temporal processing deficits in the pre-senescent auditory system. J. acoust. soc. am. 2006;119(4):2305-15.

26. Calais LL, Russo ICP, Borges ACLC. Desempenho de idosos em um teste de fala na presença de ruído. Pró-fono. 2008;20(3):147-52.

DOI: 10.1590/S1516-18462010005000078

RECEBIDO EM: 12/11/2009

ACEITO EM: 21/04/2010

Endereço para correspondência:

Flávia Duarte Liporaci

Rua Marechal Cantuária, 148/201

Rio de Janeiro - RJ

CEP: 22291-060

E-mail: flavia.liporaci@oi.com.br 